\title{
Elevated progesterone levels in women on DHEA supplementation likely represent assay interference
}

\author{
Eric J. Forman • Jason M. Franasiak • Richard T. Scott Jr.
}

Received: 21 July 2014 / Accepted: 26 January 2015 /Published online: 15 February 2015

(C) Springer Science+Business Media New York 2015

Dear Editor,

We read with interest the recent case report by Strauss et al. regarding a woman with diminished ovarian reserve who had higher serum progesterone levels after treatment with DHEA [1]. The authors clearly demonstrate that serum progesterone measurements increased while the patient was receiving DHEA supplementation after ovarian transplantation. The authors hypothesized that this progesterone must be derived from the adrenal glands. Another significant possibility exists. Data from our lab has demonstrated that increased progesterone levels seen after DHEA administration are likely due to crossreactivity with the immunoassay and do not represent circulating progesterone. Our group and others have noted that when women were placed on DHEA, baseline serum progesterone levels were markedly higher than those measured prior to DHEA supplementation [2]. While other authors have assumed this indicated a true increase in progesterone production of either an ovarian or adrenal source, the possibility of assay interference must be considered. Much of the ingested DHEA is rapidly sulfated and converted to DHEA-SO 4 . We recently demonstrated [3] that the addition of pure DHEA- $\mathrm{SO}_{4}$ solutions

E. J. Forman $(\bowtie) \cdot$ J. M. Franasiak $\cdot$ R. T. Scott Jr.

Division of Reproductive Endocrinology \& Infertility, Reproductive

Medicine Associates of New Jersey, Basking Ridge, NJ 07920, USA

e-mail: eforman@rmanj.com

E. J. Forman · J. M. Franasiak • R. T. Scott Jr. Department of Obstetrics, Gynecology \& Reproductive Sciences, Rutgers Robert Wood Johnson Medical School,

New Brunswick, NJ 08901, USA (containing no progesterone) resulted in dose-dependent increases in progesterone assay results in spite of there being no additional progesterone in the samples. This phenomenon was demonstrated on three different commercially available immunoassay platforms. Thus, the elevated progesterone measured in this young woman post-ovarian transplant likely reflects the fact that she was properly taking her prescribed DHEA and the progesterone assay cross-reacted with her elevated serum DHEA-SO $\mathrm{S}_{4}$. The implications of this assay interference go beyond monitoring of the resumption of ovarian function post transplantation. Given the importance of late follicular progesterone elevations on clinical outcomes after fresh embryo transfer [4], it is important for each clinical program that prescribes DHEA to quantify the level of interference with their progesterone assay and to consider those data when making clinical decisions.

\section{References}

1. Strauss S, Greve T, Ernst E, Fraidakis M, Grudzinkas JG, Andersen CY. Administration of DHEA augments progesterone production in a woman with low ovarian reserve being transplanted with cryopreserved ovarian tissue. J Assist Reprod Genet. 2014;31:645-9.

2. Weissman A, Horowitz E, Ravhon A, Golan A, Levran D. Dehydroepiandrosterone supplementation increases baseline follicular phase progesterone levels. Gynecol Endocrinol. 2011;27:1014-7.

3. Forman EJ, Franasiak JM, Scott K, et al. DHEA supplementation results in supraphysiologic dhea-s serum levels that interfere with progesterone $(\mathrm{P})$ immunoassays, resulting in spurious $\mathrm{p}$ elevations that may alter clinical management in IVF. Fertil Steril 2014;102(3):e306

4. Bosch E, Labarta E, Crespo J, Simon C, Remohi J, Jenkins J, et al. Circulating progesterone levels and ongoing pregnancy rates in controlled ovarian stimulation cycles for in vitro fertilization: analysis of over 4000 cycles. Hum Reprod. 2008;23:2346-51. 